\title{
El "Muro Mediterráneo" en el territorio de la Marina Alta: búnkeres y baterías de la Guerra de España (1936-1939)
}

The "Mediterranean Wall" in the territory of the Marina Alta: bunkers and batteries of the Spanish War (1936-1939)

\author{
Andrés Martínez-Medina a , Antoni Banyuls i Pérez ${ }^{\text {b }}$, Andrea Pirinu ${ }^{\mathrm{c}}$ \\ ${ }^{\text {a } U n i v e r s i t a t ~ d ' A l a c a n t, ~ A l i c a n t e, ~ S p a i n, ~ a n d r e s m . m e d i n a @ u a . e s ~}$

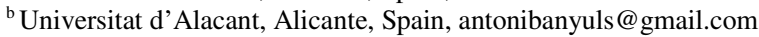 \\ ${ }^{\mathrm{c}}$ Università degli Studi di Cagliari, Cagliari, Italy, apirinu@unica.it
}

\begin{abstract}
In 1936-1939 the War of Spain took place, turning its territory into the testing ground of Europe in anticipation of the Second World War; here new weapons were tested: mass media, propaganda and aviation. The national side used Mallorca as "aircraft carrier" from which it launched airstrikes on the Mediterranean coast: a rearguard that required fortification. To defend the cities, the Republican government ordered, in 1937, to build a coastal defensive system ("Mediterranean Wall"). On the Valencian coast there were ten basic enclaves: from the lighthouse of Castellón to the end of Santa Pola. This network of defenses had two built lines. The first was constituted by elements located at zero level, by the sea and on the beaches, which maintained regular distances from each other; these were reinforced concrete bunkers that sought to camouflage themselves. A second was formed by coastal and antiaircraft, concrete and masonry batteries that merged with the land, located in the hills to have a wider horizon and be closer to its objectives. Bunkers and batteries that followed geometric patterns in constant evolution.

This communication studies the defensive settlements built by the Republican army in the cities of Xàbia and Dénia (Marina Alta), which had a port, airfield and armament factories, which made them the target of enemy aviation. In these territories many of these architectures have disappeared under real estate pressure, but there are still several bunkers, batteries and ammunition deposits that are intended to be inventoried and documented (especially the $7^{\text {th }}$ of the Montgó and the $8^{\text {th }}$ of the Portixol batteries) to insert into of the tradition of historical military forts (typological genealogies) and their understanding as a networked defensive system that maintains parallels with the system of coastal towers of the system of coastal towers of the Modern Age.
\end{abstract}

Keywords: Mediterranean wall, bunkers and batteries, modern defenses, reinforced concrete.

\section{Crónica anticipada de una guerra: mass media, publicidad y aviación}

Durante el periodo de 1936 a 1939 tuvo lugar la Guerra de España, transformando su territorio en el laboratorio de ensayo del teatro bélico de Europa al adelantarse a la II Guerra Mundial. En la península ibérica se testaron tres nuevas armas que ensancharon la confrontación más allá del campo de batalla: los mass media, la publicidad bélica y el armamento mecanizado por tierra, 


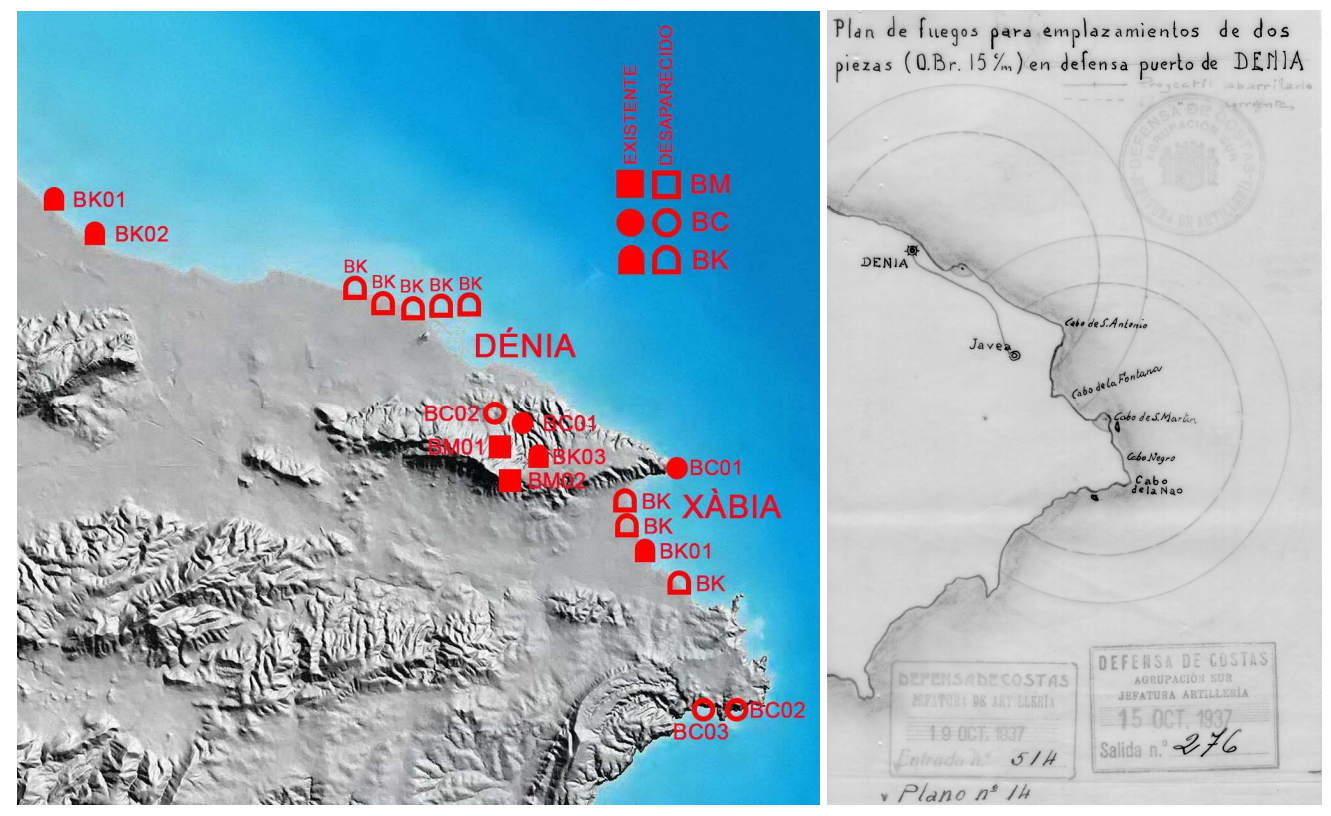

Fig. 1. Izq: Situación de las defensas en los términos de Dénia (den) y Xàbia (jav) en plano de relieve de la comarca, señalando los existentes y los demolidos. Fig. 2. Dcha: Fuegos de las baterías de Dénia y Xàbia (Larios 2019).

mar y aire. De este modo, los frentes a atacar y resguardar no serían tan exactos como antaño, sino que fueron más amplios e imprecisos. Entre los medios de comunicación de masas que prestaron un rol protagonista destacan la prensa, el cine y la radio; sin entrar en los detalles de la manipulación de los diarios, ni en la orientación ideológica de muchos filmes -el cine se erigiría en el pasatiempo visual preferido por las masas, el cual se dotaría en los años previos al conflicto del color y el sonido-, conviene apuntar que la radio, que debutó ante el gran público en 1920, tuvo un éxito clamoroso y, hacia 1930, ya había emisoras por todo el planeta y enseguida tuvo su aplicación militar como canal de comunicación en los diferentes ejércitos. Por lo que respecta a la publicidad política, los carteles se estamparon para campañas de propaganda a fin de elevar la moral de las propias tropas que despreciaban al adversario y exageraban su capacidad ofensiva e industria bélica, alentando la solidaridad para levantar refugios y defensas, entre otros. Estos pósteres, muchas veces, denotaban una fuerte influencia de las vanguardias arquitectónicas y artísticas de entre guerras (del futurismo al artdecó), ensayando con fotomontajes y collages.
Pero sería el nuevo armamento mecánico el que más modificaría la defensa de las fronteras ya que, al tratarse de artefactos móviles, obligaba a emplear las construcciones defensivas de manera dispersa por los frentes que se esperaba pudiese llegar el adversario, fuese por tierra, mar o aire. Por ello, la aviación sería decisiva a la hora de diseñar una estrategia que pudiese detener o repeler al enemigo desde posiciones fijas que, en la medida de lo posible, deberían pasar lo más desapercibidas, cuando no permanecer ocultas. Cierto que en España se probaron aviones y tanques alemanes y rusos, pero, ante la hipótesis de un desembarco en las costas mediterráneas, sería el uso por parte del bando nacional de la isla de Mallorca como un 'portaviones', desde el que se lanzaron continuas incursiones aéreas en la zona republicana, lo que exigió la fortificación de su retaguardia litoral. Por lo que respecta a la Marina Alta, con dos destacamentos de baterías (Fig. 2) las ofensivas aéreas comenzaron el 13 de agosto de 1937 y acabaron el 28 de marzo de 1939, sumándose más de 30 días de bombardeos (Aracil y Villaroya, 2010). 
Para defender las ciudades, desde la capital provisional de Valencia, el gobierno republicano ordenó, en 1937, construir un sistema de fortificaciones por todo el litoral a erigir junto a las infraestructuras portuarias y las ensenadas propicias a un desembarco (Gil, Galdón, 2007); a esta línea intermitente de defensas la denominamos "Muro Mediterráneo" por su parecido con la estrategia -salvando la escala y las condiciones de acabado material- del Atlantik Wall que erigiría la organización TODT un lustro después desde Normandía hasta Noruega (Martínez-Medina, Sanjust, 2013). En la costa valenciana se previeron inicialmente cinco enclaves: Castellón, Sagunto, Valencia, Denia-Jávea y Alicante, red que se ampliaría con más destacamentos a medida que avanzaba el conflicto (Canet, Sagunto, El Puig, Cullera), si bien no serían estas las únicas poblaciones protegidas. Este sistema de defensas estaba constituido por dos líneas fuertes sitas a diferente nivel. Una primera estaba formada por una serie de elementos enclavados a cota cero, junto al mar y sobre las playas, emplazados a distancias repetidas entre sí que estaban en función del alcance de tiro; se trataba de una línea de búnkeres de hormigón, con diversas soluciones en atención al armamento con el que contaban, diseñados con uno o dos senos según incorporaran una o dos ametralladoras. Y una segun- da, más estratégica, compuesta por una serie de baterías de costa y antiaéreas, ejecutadas en mampostería y hormigón, que se ubicada en las colinas, para disponer de un horizonte más amplio y estar más cerca de los aviones.

En el presente artículo se estudian las defensas erigidas por la II República en las ciudades de Dénia y Xàvia (Fig. 1), en la costa norte de la provincia de Alicante (incluyendo las baterías $7^{\mathrm{a}}$ y $\left.8^{\mathrm{a}}\right)$, que contaban con puertos, aeródromos y fábricas de armamento, lo que las convirtió en objetivo de la aviación fascista. En estos lugares, a lo largo de las ocho décadas transcurridas, han desaparecido muchas de estas construcciones bajo la presión turística y la desidia general por lo incómodo de este patrimonio, pero aún quedan en pie restos de búnkeres, alguna batería y otras piezas por documentar (incluyendo cierta información respecto de los ya desaparecidos) para insertar estas arquitecturas militares dentro de la cadena evolutiva de las defensas históricas (genealogías tipológicas, desarrollo geométrico) y su comprensión como un sistema dispuesto en red para su eficacia táctica. Sumamos pues este trabajo a otros anteriores que han registrado los restos de las defensas de la guerra civil en esta zona y limítrofes (Ferrer, Ferrer Hermenegildo, 2000; Bolufer, 2013; Larios, 2019).

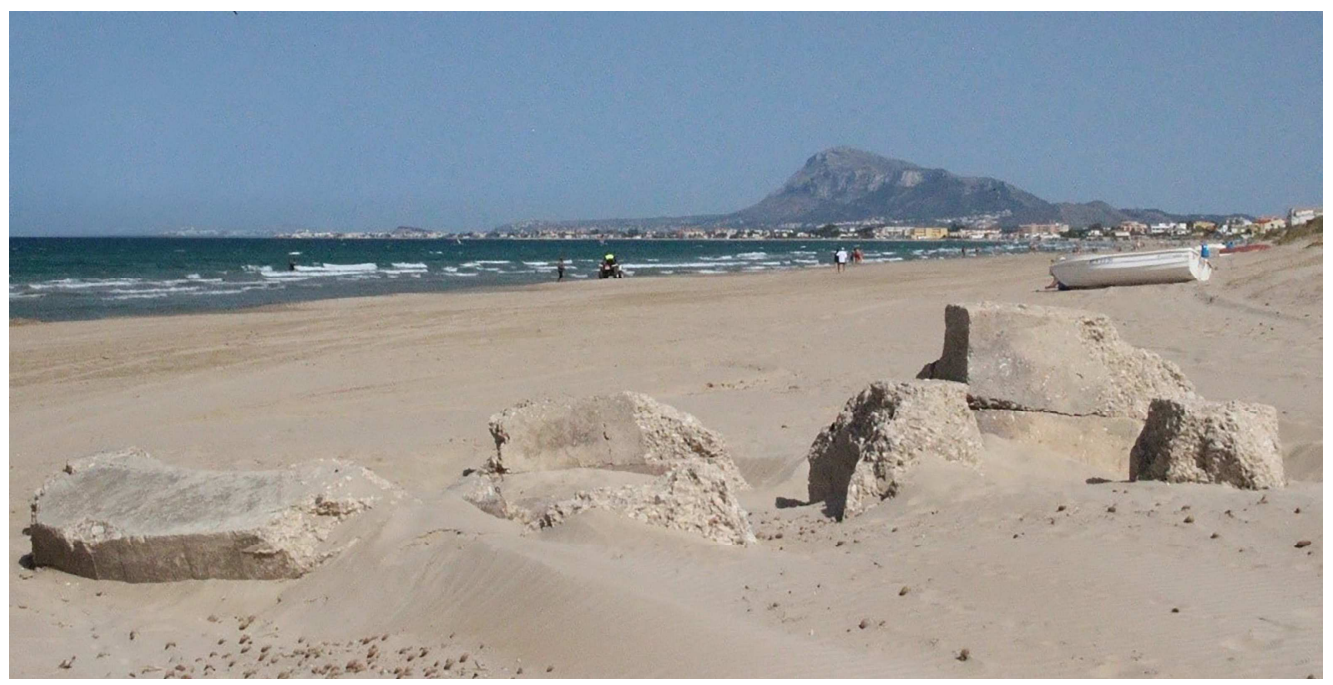

Fig. 3. Restos de uno de los búnkeres sito en playa de Les Marines de Dénia (BK02.den) (Fuente propia, 2016). 


\section{Las baterías de costa y otras defensas en la comarca de la Marina}

En un estudio de inventariado de los búnkeres, baterías y barracones vinculados al sistema de defensas del litoral en la provincia de Alicante (Martínez, 2016) se establecen siete zonas. La primera de ellas, por el norte, se la denomina Costa Norte $(\mathrm{CN})$ y abarca desde Els Poblets (antes Setlla, Mirarosa y Miraflor), pasando por Dénia (Denia), Xàbia (Jávea), Poble Nou de Benitaxell (Benitachel), el par Teulada-Moraira, Benissa (Benisa), Calp (Calpe), Altea, Benidorm y La Vila (Villajoyosa). Solo en cuatro de estos poblados -Dénia, Xàbia, Altea y Benidorm- se sabe de la presencia de defensas fijas. Se trata de arquitecturas desplegadas a los dos lados del núcleo urbano y su puerto y que conformaron un modelo de fortificación aplicado a muchos municipios, mediante la disposición de búnkeres a ras de playa jalonando la costa a intervalos de 500-750 m de media, tratando de salvar diques portuarios, estuarios fluviales y la navegación de cabotaje. Cuando las ciudades acogían en su término otras instalaciones, la red se reforzaba con baterías de costa situadas a cierta cota y ocultas. Ambas líneas de defensa están presentes en la Marina Alta en un estado muy deteriorado, reintegrándose sus elementos, poco a poco, a la naturaleza con la que, a veces, se mimetizaron.

\subsection{Dénia: su frente litoral y el monte Montgó}

La ciudad y el término de Dénia, por su relieve estratégico y su intendencia militar para el ejército republicano por las fábricas de juguetes y otros enseres que fueron reconvertidas con fines bélicos, sufriría un total de 32 bombardeos en los tres años de contienda por parte de la aviación italiana con base en las Islas Baleares, causando medio centenar de víctimas mortales (Aracil, Villarroya 2010). Todo ello a pesar de que se contaba con varios refugios antiaéreos para protección de la población distribuidos por distintos sitios de la trama urbana y de los cuales se conserva el túnel bajo el castillo.

En el término municipal se tiene constancia de la existencia en su momento de hasta un total de, al menos, unos sietes búnkeres, desplegados desde el río Girona hasta el brazo norte del puerto, donde se extiende un conjunto de playas y costa baja de varios kilómetros (Les Marines, El Raset y el Marge Roig). De toda esta línea de defensa junto al mar, hoy solo se conservan ruinas de dos búnkeres (BK01.den y BK02.den), de difícil identificación, esparcidas como los restos de un naufragio por la arena (Fig. 3); de hecho, serían necesarios trabajos arqueológicos para definir su inicial geometría. También se descubren fragmentos de otro fortín junto a la escollera, mientras que otros cuatro búnkeres han sucumbido bajo las urbanizaciones de todo este frente. Todos estos búnkeres estaban ejecutados en hormigón armado como los fortines de Xàbia y Altea $\mathrm{y}$, por las fotos de época, responden a patrones tipológicos de uno o dos senos con una geometría elemental, racional y funcional, y estaban previstos y provistos para una o dos ametralladoras en correspondencia.

A diferencia de la línea de búnkeres, sí se ha mantenido en pie el conjunto defensivo formado por una batería antiaérea y de costa (BC01.den), el refugio anexo (BM01.den), restos del búnker de telemetría (BK03.den) y el polvorín de suministro de municiones (BM02.den), sito en las faldas de la colina Montgó al sur del término municipal. Es probable que este enclave se completase con una segunda batería a escasos $200 \mathrm{~m}$ de la existente, si bien puede que hoy esté convertida en piscina de una vivienda. Aunque este enclave ya ha sido estudiado (Ferrer, Ferrer Hermenegildo, 2000), conviene citar algunos datos más. Se trata del destacamento de costa proyectado para Dénia que, ya en octubre de 1937, se preveía dotarlo con dos piezas obuses de bronce de $150 \mathrm{~mm}$ (Cabezas, 2017). Este asentamiento se conformaba con dos baterías circulares de las que permanece una con rampa de acceso, de mampostería, con $7,10 \mathrm{~m}$ de $\varnothing$ y dos habitáculos laterales para munición y resguardo (Fig. 4); su geometría se vincula a la cadena tipológica que la relaciona, tanto con la batería del Saler, como con la del cabo de Huertas de Alicante (la mayor de las presentes en este litoral con $15,00 \mathrm{~m}$ de $\varnothing$ ) y con las del cabo de Santa Pola $(12,00 \mathrm{~m}$ de $\varnothing)$.

Las baterías requerían de dos construcciones más para su función: el búnker-telémetro para el 


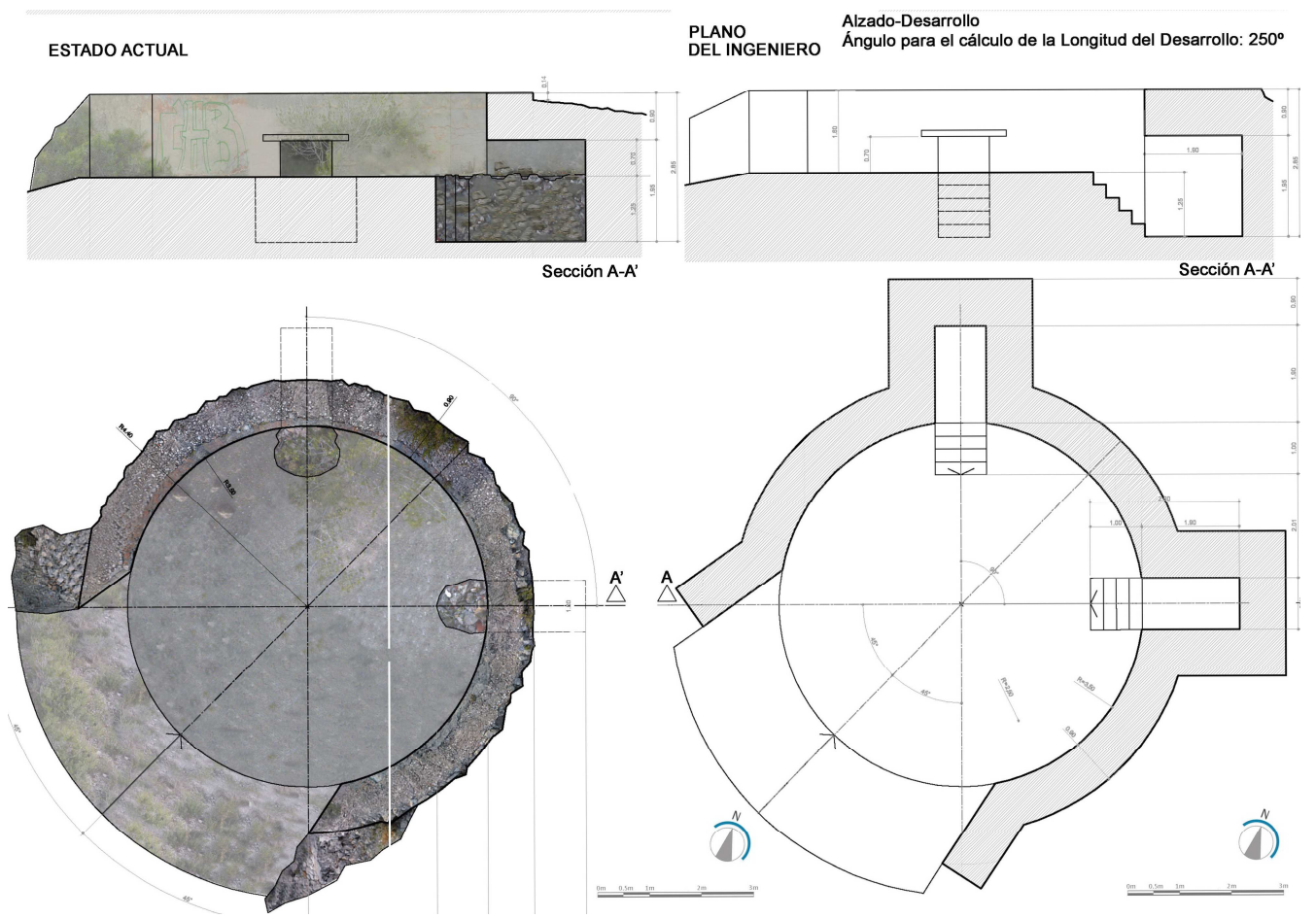

Fig. 4. Alzamiento batería destacamento de Dénia (BC01.den), por E. Casassola, S. Vilella y D. Villacís (2012).

cálculo de tiro y el polvorín, soterrado para proteger la munición almacenada que se extraía mediante un sistema de elevación vertical. Se completaba el conjunto con un refugio semienterrado para escondite de los soldados del destacamento en caso de ataque aéreo (Fig. 5), que consiste en un túnel alargado, de sección trapezoidal, cubierto con una bóveda rebajada, con accesos por escalera en los dos extremos y pequeños huecos de aireación en la parte superior para ventilar la estancia; este refugio respondía a patrones estandarizados de catálogos de los zapadores ya que se construirían en varios lugares de España e Italia, años después. La residencia de los soldados se preveía en las casas próximas, ya que el refugio lo era solo para caso de emergencia (Cabezas, 2017).

\subsection{Xàbia: su costa y el cabo Sant Martí}

La ciudad de Xàbia, a pesar de disponer de un puerto comercial, fue bombardeada solo una vez (Aracil, 2010); no obstante, disponía de cinco refugios soterrados para la población civil: cuatro se emplazaban bajo espacios públicos y un quinto en la ladera junto al puerto, en forma de galería excavada. En el término municipal se encontraba el aeródromo del Plà, con dos pistas de despegue (de ca. 1000 x 570 m y 920 x 200 m), apisonadas en 1937 y usadas en pocas ocasiones; en sus inmediaciones (finca Rebaldí) se erigía el pabellón para los pilotos y el personal auxiliar, así como un refugio enterrado (ya desaparecido) de 3,40 x 6,80 m (Bolufer, 2013); los restos de este conjunto se encuentran en propiedad privada. De modo similar a Dénia, Xàbia tenía dos líneas de defensas, una bordeando la costa a nivel del mar y otra en altura en el entorno del cabo de Sant Martí, donde se ubicaba el destacamento de costa. Por la presión inmobiliaria, muchas de estas defensas han desaparecido o, al encontrarse algunas en parcelas privadas, si se conservan, se ignora su estado de conservación.

El primer frente de defensas estaba compuesto por unos cuatro búnkeres separados, a distancias fijas, desde el cabo de Sant Antoni hacia la playa del Arenal. De esta ristra de fortines se tienen 

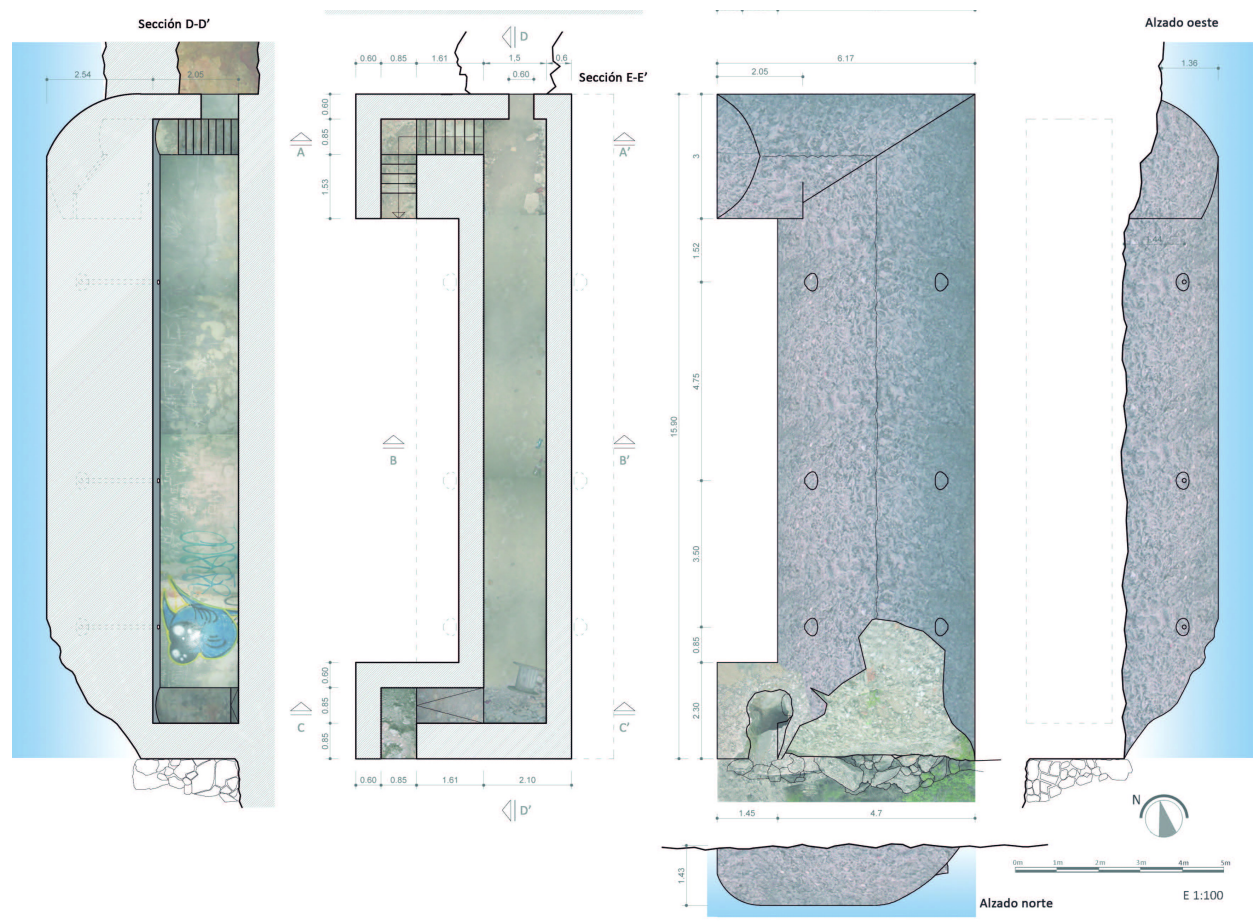

Fig. 5. Alzamiento refugio destacamento de Dénia (BM01.den), por E. Casassola, S. Vilella y D. Villacís (2012).

instantáneas de antaño. El primer búnker documentado -búnker del moll- se emplazaba en el inicio de la escollera sur del puerto. Por las fotografías, parece responder al mismo patrón geométrico que los otros: constaba de dos senos con un remate superior a dos aguas. El segundo búnker yacía en la playa del Benissero, a unos 500 $\mathrm{m}$ a mediodía del anterior; presentaba dos senos con tres aspilleras cada uno en su frente barriendo $180^{\circ}$, estaba hecho de hormigón y contaba con una cubierta troncocónica. Un tercer búnker (BK01jav) se disponía en la zona de roca baja del Montanyar, a unos 500 m más al sur; este sería demolido (ca. 1998), si bien aún resta su huella en la costra pétrea que muestra el tipo de dos senos, con tres aspilleras cada uno, levantado en hormigón recubierto de pequeñas piedras tratando de camuflarse con el medio. Y el último y cuarto fortín, también destruido, se hallaba muy cerca de la playa del Arenal, a unos 500 m aún más al sur, cerca del actual Parador. En las fotografías de archivo se aprecia que era de dos senos con tres aspilleras y acceso por paso con bóveda desde la parte posterior sita a poniente; te- nía pegadas lajas de piedra tosca en el techo de hormigón. El sistema lineal de estos búnkeres, erigidos en hormigón armado con una capa final de piedras para su mimetización, responde a idénticos tipos geométricos de dos senos, previstos para dos ametralladoras. Al margen de este cuarteto ya desaparecido, se encuentra un nido circular, a modo de pequeña batería a cielo abierto (BC01jav) de dimensiones mínimas (2,00 m de Ø), emplazado al borde del acantilado junto a la estación de señales del siglo XIX en lo alto del cabo de Sant Antoni, a 150 m.s.n.m. El antepecho circular de cierre está levantado con mampostería y tiene un rebaje en su parte frontal para apoyar el armamento. Por sus dimensiones es difícil que sirviera de batería de costa; tal vez funcionó como puesto de observación, dada su posición en altura, cubriendo a las propias instalaciones del faro.

En cuanto a las baterías parece que se manejaron dos posibles lugares en el entorno del cabo de Sant Martí, en el paraje del Portixol. El más seguro se dispone en terrenos particulares, estaría 


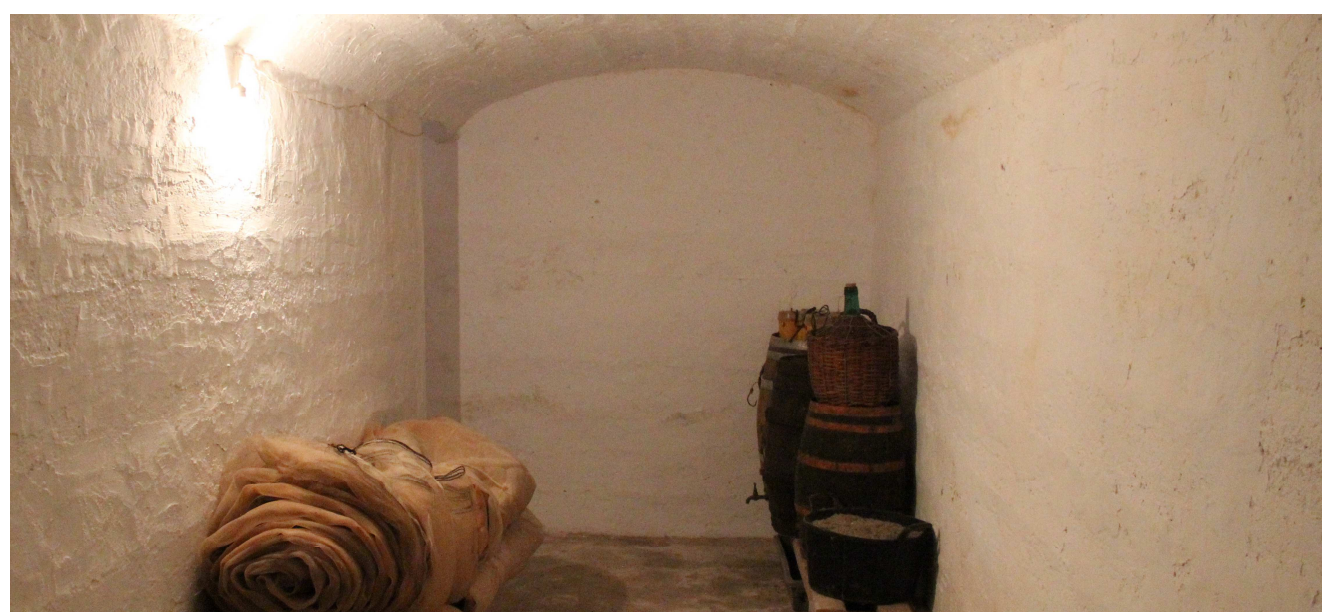

Fig. 6. Interior del refugio del destacamento de Xàbia en su estado actual, privado (Martínez-Medina, 2016).

compuesto por dos baterías, un polvorín y un refugio, y debía contar con un búnker telémetro. Las baterías se situaban a unos 90 m.s.n.m., de $10,00 \mathrm{~m}$ de $\varnothing$, si bien, en las fotografías aéreas de 1961 solo se localiza la huella de una, tal vez porque la otra yace bajo un inmueble turístico (Bolufer, 2013). El enclave tiene un refugio semienterrado (Fig. 6), de perímetro rectangular (ca. 12,00 x 2,00 m) con acceso por un par de escaleras en sus extremos, está construido en hormigón armado (pueden verse los listones del encofrado) y cubierto por una bóveda rebajada. con muros de perfil inclinado al exterior con un relleno de hormigón $(+1,50 \mathrm{~m})$ y respiraderos; muy cerca se localiza un polvorín al que se entra a través de un túnel. Ninguno de estos dos elementos - refugio y polvorín - se han podido dibujar en su estado actual, al encontrarse dentro de fincas privadas. Este destacamento coincide con el previsto por el ejército republicano en el último trimestre de 1937 para colocar las baterías en el Portixol con dos obuses de bronce de $150 \mathrm{~mm}$ (Cabezas, 2017). Así pues, esta defensa se asemejaría a la del Montgó, ya que ambas conforman las baterías $\mathrm{n}^{\circ} 7$ en Dénia y $\mathrm{n}^{\circ} 8$ en Xàbia de la Agrupación Sur de Defensa de Costas en noviembre de 1937 (Cabezas, 2017).

\section{El incómodo patrimonio de las guerras}

Efectuado este repaso a las defensas existentes en Dénia y Xàbia, verificamos que eran muy similares. Ambas ciudades presentaban dos líneas de defensa: una primera de búnkeres a nivel del mar (en la costa norte de Dénia y al sur del puerto de Xàbia), que cubrían la navegación de cabotaje y el litoral ante un desembarco, y una segunda línea de destacamentos de costa, sitos en cotas altas que, constituidos cada uno por un par de baterías, un refugio, un polvorín y un búnker de telemetría, se equipaba con obuses. De todo ello solo quedan restos de búnkeres en las playas de Dénia, el asentamiento incompleto en las faldas del Montgó (Dénia) y los restos del destacamento del cabo de Sant Martí, Portixol (Xàbia), ya sin baterías. Todos estos elementos arqueológicos vienen recogidos en los catálogos de los planes urbanísticos.

Este inventario de bienes vinculados a la Guerra de España de 1936-1939 no es asimilable a la versión clásica del patrimonio, más bien, aquí se recibe una herencia incómoda por el contexto político bajo el cual se materializó. Se trata de arquitecturas que responden a patrones casi de producción industrial difíciles de encuadrar con el concepto estereotipado de monumento que, además, se sitúan en lugares de difícil acceso. Los búnkeres $-\mathrm{y}$ en menor medida las baterías y los refugios- representan, además, objetos abominables desde su concepción y cualquier intento de redención tropieza siempre con su función inicial: la del ingenio militar fabricado para matar. A negative monument, a fearsome heritage (monumento negativo, patrimonio temible), que apuntan Cocroft y Schofield (2009), y el discord 
value (valor de discordia), que señala Dolff (2001), son términos para referir el legado físico de los conflictos de los siglos XX y XXI que ponen en evidencia la dificultad de encaje de este patrimonio en el discurso hegemónico. Nos situamos ante un legado masivo y repetido por la geografía española (de uno y otro bando) que difícilmente construye identidad de igual modo en la sociedad, por lo que su preservación no resulta inmediata. Sin embargo, cabría pensar -al margen del riguroso inventario de los restos para el conocimiento del pasado- en la vía de recuperar el destacamento mejor mantenido y preservarlo para el futuro en tanto que pieza clave de un "paisaje de las guerras modernas", casi al mismo nivel que hemos protegido a las torres vigías renacentistas. Quizás podríamos elegir el destacamento en el Montgó (Dénia), que cuenta con cuatro piezas (batería, telémetro, polvorín y refugio), proceder a su restauración y acondicionar la zona para hacerla más accesible de manera que resulte fácil su entendimiento. Esta intervención se completaría con visitas organizadas desde la Oficina de Turismo. De esta manera, se preservaría el único enclave que, casi en su totalidad, nos ha llegado al presente, se daría una información del sistema de las defensas de la retaguardia republicana (que luego el gobierno de Franco ampliaría en otros frentes) y se consolidaría un paisaje de las ruinas de las guerras cuyo juicio ya no nos corresponde porque, tarde o temprano, todo el dolor pasará al olvido para nuestra convivencia pacífica como siempre ha sido (Rieff, 2017).

\section{Bibliography}

Aracil, R.; Villarroya, J. (2010). El País Valencià sota les bombes (1936-1939), UPV, València.

Bolufer Marqués, J. (2013). "L'arquitectura de la guerra a Xàbia”, in Festes Patronals de la Mare de Déu de Loreto, Ajuntament de Xàbia Ed., Alacant, pp. 43-47.

Cabezas Sánchez, A. (2017). “La artillería de costa en Levante durante la guerra civil, 1936-1939”, (capítulo para un libro en prensa a cargo de José María Manrique).

Cocroft, W.; Schofield, J., eds. (2009). A fearsome heritage: diverse legacies of the Cold War, Left Coast Press, Walnut Creek.

Dolff-Bonekämper, G. (2001). "Sites of memory and sites of discord: Historic monuments as a medium for discussing conflict in Europe", in Forward planning: the function of cultural heritage in a changing Europe, Consejo de Europa.

Ferrer, A.; Ferrer Hermenegildo, V. (2000). "Arquitectura Militar del s. XX a Dénia", Aguaits, 17, in https://www.raco.cat/index.php/Aguaits/article/view/66170/85605.

Gil Hernández, E.R.; Galdón Casanoves, E. (2007). “Arqueología valenciana de la Guerra Civil”, in VV.AA., La Guerra Civil en la Comunitat Valenciana. 17: El Patrimoni material, Prensa Valenciana, Barcelona.

Larios, V. (2019). "La 7 $7^{a}$ Batería de la Agrupación Sur de Defensa de Costas", in Seser, R. ed., Fem Memòria: Dénia, guerra i represió, Ajunt. Dénia i IECMA, Alacant, pp. 80-86.

Martínez-Medina, A., dir. (2016). Arquitecturas para la defensa de la costa Mediterránea (1936-1939), Universidad de Alicante, Alicante, in http://hdl.handle.net/10045/81287.

Martínez-Medina, A.; Sanjust, P. (2013). "Muro Mediterráneo versus Movimiento Moderno", I2, 1, in https://doi.org/10.14198/i2.2013.1.02.

Rieff, D. (2017). Elogio del Olvido, Debate Ed., Barcelona. 